\title{
Beyond Spatial Memory: The Anterior Thalamus and Memory for the Temporal Order of a Sequence of Odor Cues
}

\author{
Mathieu Wolff, Sheree J. Gibb, and John C. Dalrymple-Alford \\ Van der Veer Institute for Parkinson's and Brain Research and Department of Psychology, University of Canterbury, Christchurch 8020, New Zealand
}

Influential recent proposals state that the anterior thalamic (AT) nuclei constitute key components of an "extended hippocampal system." This idea is, however, based on lesion studies that used spatial memory tasks and there has been no evidence that AT lesions cause deficits in any hippocampal-dependent nonspatial tasks. The present study investigated the role of the AT nuclei in nonspatial memory for a sequence of events based on the temporal order of a list of odors, because this task has recently been shown to depend on the integrity of the hippocampal formation. After preoperative training, rats with excitotoxic lesions of the AT nuclei showed a severe and selective postoperative impairment when required to remember the order of pseudorandom sequences of six odors. The rats with AT lesions were able instead to learn two new tasks that required recognition memory and the identification of the prior occurrence of events independent of their order. These results strongly matched those described after hippocampal lesions and provide the first unequivocal evidence of a detrimental effect of an AT lesion on a nonspatial hippocampal-dependent memory task.

Key words: anterior thalamic nuclei; memory for temporal order; nonspatial memory; rat; odor; hippocampus

\section{Introduction}

Disruption of the anterior thalamic (AT) nuclei in humans is associated with severe anterograde amnesia (Harding et al., 2000; Van der Werf et al., 2000). Because extensive neural connections exist between the AT nuclei and the hippocampal system, these observations have generated influential proposals that the AT nuclei are a critical nodal point in an "extended hippocampal system" (Aggleton and Brown, 1999; Vann and Aggleton, 2004). This idea is supported by growing evidence that, like hippocampal system lesions, AT lesions produce profound impairments in various spatial memory tasks (Aggleton et al., 1996; Byatt and Dalrymple-Alford, 1996; Parker and Gaffan, 1997; Sziklas and Petrides, 1999; Warburton and Aggleton, 1999; Warburton et al., 1999; van Groen et al., 2002; Mair et al., 2003; Mitchell and Dalrymple-Alford, 2005).

In contrast, there are virtually no indications that AT lesions also impair memory in nonspatial tasks, as assessed by spontaneous object recognition memory, configural learning, or sensory preconditioning, but these tasks are now acknowledged as generally insensitive to, or have produced mixed findings after, hippocampal lesions (Warburton and Aggleton, 1999; WardRobinson et al., 2002; Moran and Dalrymple-Alford, 2003). One interesting exception is that AT lesions may impair go/no-go delayed alternation tasks by producing a delay-dependent deficit

Received Dec. 21, 2005; revised Jan. 18, 2006; accepted Jan. 28, 2006.

This work was supported by a University of Canterbury (New Zealand) research grant. We thank Silvana de Freitas Costa and Melissa Adams for assistance in behavioral testing.

Correspondence should be addressed to John C. Dalrymple-Alford, Van der Veer Institute for Parkinson's and Brain Research and Department of Psychology, University of Canterbury, Private bag 4800, llam Road, Christchurch 8020, New Zealand. E-mail: john.dalrymple-alford@canterbury.ac.nz.

DOI:10.1523/JNEUROSCI.5481-05.2006

Copyright $\odot 2006$ Society for Neuroscience $\quad 0270-6474 / 06 / 262907-07 \$ 15.00 / 0$
(Beracochea et al., 1989; Peinado-Manzano and Pozo-Garcia, 1991; Celerier et al., 2000), but the nonspatial characteristic of some of this evidence has been questioned (Ward-Robinson et al., 2002). Moreover, there is uncertainty whether hippocampal lesions reliably impair the mnemonic characteristic of delayed alternation tasks (Winocur, 1985; Foster and Rawlins, 1992).

Probably the strongest evidence that the influence of the hippocampal system on memory extends beyond the spatial domain is that it plays a major role in temporal processing, particularly memory for the temporal order of events. The separation of closely linked events across time may be a crucial aspect of memory for unique behavioral episodes. Initially, the hippocampus was shown to support temporal order memory for sequential spatial locations (Chiba et al., 1994; Kesner, 1998), but recent studies have found that the hippocampus is also important for temporal order memory of nonspatial items. Using slightly different procedures, Fortin et al. (2002) and Kesner et al. (2002) found that rats with hippocampal lesions were severely impaired when required to judge the relative position of two items derived from a recently presented list of five odors. In contrast, the hippocampus does not mediate memory for simple odor information or the relative novelty of an odor (Dudchenko et al., 2000; Fortin et al., 2002). The current study, then, determined whether the AT nuclei are similarly involved in temporal order memory of nonspatial items based on the presentation of a sequence of odors. Performance on this task was compared with that on two tasks that tested, respectively, matching-to-sample and nonmatching-to-sample recognition memory for a list item versus a nonlist item. It was hypothesized that AT rats would be impaired in the temporal order memory task but would be able to learn recognition memory tasks, because the latter do not require the retention of a list of sequential events. 
Table 1. Coordinates and infusion rates used for each injection site for the NMDA lesions

\begin{tabular}{llr}
\hline & AT & \\
\cline { 2 - 3 } & Ant & Post \\
\hline AP coordinates for B-L distance & & \\
$(\mathrm{cm})$ & & \\
$0.60-0.61$ & -0.23 & -0.24 \\
$0.62-0.63$ & -0.24 & -0.25 \\
$0.64-0.66$ & -0.25 & -0.26 \\
$0.67-0.72$ & -0.26 & -0.27 \\
ML distance & \pm 0.120 & \pm 0.150 \\
DV distance & -0.58 & -0.555 \\
Volume $(\mu l, 0.12 \mathrm{M})$ & 0.09 & 0.11 \\
Infusion rate $(\mu \mathrm{l} / \mathrm{min})$ & 0.03 & 0.03 \\
\hline
\end{tabular}

Anterior (Ant) and posterior (Post) coordinates; AP varied with bregma (B) to lambda (L) distance.

\section{Materials and Methods \\ Subjects}

Female PVGc hooded rats, bred in-house and with an initial weight of $180-220 \mathrm{~g}$ ( $\sim 10$ months old at the beginning of the study), were housed in groups of three or four in opaque plastic cages $(27 \mathrm{~cm}$ by $45 \mathrm{~cm}$ wide by $22 \mathrm{~cm}$ high) under a reversed light schedule (off 8 A.M. to 8 P.M.). Testing occurred during the dark portion of the cycle. Rats had access to water ad libitum and were maintained at $80-85 \%$ of ad libitum weight, bar food access ad libitum just before and after surgery to facilitate postoperative recovery. All protocols conformed to the National Institutes of Health Guide for the Care and Use of Laboratory Animals and were approved by the Animal Ethics Committee of the University of Canterbury.

\section{Surgery}

Only rats that reached criterion on the memory for temporal order task received either AT lesions $(n=11)$ or sham surgery (SHAM; $n=12$; needle lowered into the cortex but no infusion). Anesthetized rats (50 $\mathrm{mg} / \mathrm{ml}$ pentobarbitone at $1.65 \mathrm{ml} / \mathrm{kg}, 20 \mathrm{~min}$ after $0.13 \mathrm{mg} / \mathrm{ml}$ atropine at $1.5 \mathrm{ml} / \mathrm{kg}$, i.p.) were placed in a stereotaxic apparatus with the incisor bar set $7.5 \mathrm{~mm}$ below the interaural line to minimize or avoid fornix injury. After craniotomy, AT lesions were made using microinfusions of $0.12 \mathrm{M}$ NMDA (Sigma, Sydney, Australia) dissolved in phosphate buffer, $\mathrm{pH}$ 7.20 , via a $1 \mu$ l Hamilton syringe connected to a motorized infusion pump. The needle remained in situ for 3 min after each injection to allow for diffusion (for details and coordinates, see Table 1).

\section{Apparatus}

Throughout testing, single rats were placed in adjacent wood boxes ( 28 $\mathrm{cm}$ wide by $32 \mathrm{~cm}$ deep by $27 \mathrm{~cm}$ high) where they were presented with dry odorized sand in small black-painted terracotta pots $(6 \mathrm{~cm}$ inner diameter at the top by $6 \mathrm{~cm}$ high) in which they could dig for a food reward. The wire-mesh floor inside the box raised the rat above a sawdust-covered floor. Eighteen powdered odors were used each day: cloves, curry powder (each $0.5 \% \mathrm{wt} / \mathrm{wt}$ with the sand); cinnamon, garlic, ginger, onion, beef, nutmeg (each $0.8 \%$ ); basil, chives, coffee, cocoa, mint, paprika, thyme, oregano (each $1 \%$ ); parsley (2\%); cumin (5\%). A $27 \mathrm{~cm}$ wide by $6.5 \mathrm{~cm}$ high drawer was situated at the front of each box and was used to present the odor stimuli to the rat, by means of a $10 \mathrm{~cm}$ deep tray behind the front panel of the drawer such that the tray was inserted into the front of the box when the drawer was closed. The tray had three equally spaced receptacles, or holes, which were $6 \mathrm{~cm}$ in diameter, used to hold one or more pots of odorized sand, the surfaces of which were $\sim 5 \mathrm{~cm}$ above the wire-mesh floor. The pots were placed in either the central receptacle (sample phase) or in any two of the three receptacles (test phase), during which time only the box lid was open (light level, 18 lux inside the box).

\section{Behavioral testing}

Memory for temporal order. The procedure was modified from that described by Fortin et al. (2002). All rats were given three trials daily, $5 \mathrm{~d}$ per week. Each trial consisted of a sample phase of a unique (for that day's trial) pseudorandom series of six odorized sand cues, followed by a test phase in which two of the sample cues were presented and the earlier item in the list was rewarded. For the sample phase, each cue was presented singly (central receptacle), at a rate of about one odor every $3 \mathrm{~min}$, followed 3 min later by the test phase. The time between successive sample odors was the time required to present an odor cue to each of 12 rats tested in the same squad. Each sample odor was rewarded by a Sanitarium Honey Puffs cereal buried $\sim 2 \mathrm{~cm}$ below the surface of the sand (rats had previously been shaped to dig in the pots). In the test phase, two of the previous six odors were presented simultaneously; the rat had to choose the odor that had occurred earlier in the sequence to obtain an additional reward, which was now a whole Froot Loop. A choice was defined by two or more digging strokes with an anterior paw (sniffing was not considered as a choice) and rats were allowed to dig in the correct pot if they initially made an incorrect choice. One, two, or three intermediate sample items (i.e., odors) determined the temporal separation between the two probed items in the test phase, providing the three daily lag test trials, labeled lag 1, lag 2, and lag 3, respectively. Each day, each kind of trial (i.e., lag 1, 2, or 3) was used once in a pseudorandom order that varied across days, with a $1 \mathrm{~h}$ break between successive trials. For the test phase, attention was paid to ensure that no spatial bias was introduced by using a pseudorandom sequence to counterbalance the position of the rewarded and nonrewarded odors across each of the three available receptacles across trials. Because the three daily trials involved six odors each, the 18 odors were used only once per day, with the odors varying pseudorandomly in temporal position across any type of trial and across days, plus each odor was presented approximately equally as rewarded and nonrewarded odors during the test phase throughout the experiment.

We used a list of six odors instead of five odors, which had been used in previous studies (Fortin et al., 2002; Kesner et al., 2002), to ensure an equal number of different probes (test pair of odors) for each temporal separation that was used. Five odors in the sample phase permits only one possible probe in the test phase for a lag 3 trial ( 3 items, B, C, and D, intervening in the sample list between the two cues used in the probe) so that the test phase must include only the potentially easier first versus last item $(\mathrm{A}+/ \mathrm{E}-)$. With six odors in the sample series, two possible probes are available for the lag 3 trials and each probe can use only the initial or the last item for each test pair $(\mathrm{A}+/ \mathrm{E}-$ and $\mathrm{B}+/ \mathrm{F}-)$. Because there were two different probes for lag 3 trials, we also used only two probes for lag $1(\mathrm{~A}+/ \mathrm{C}-$ and $\mathrm{D}+/ \mathrm{F}-)$ and lag 2 trials $(\mathrm{B}+/ \mathrm{E}-$ and $\mathrm{C}+/ \mathrm{F}-)$, allowing an equal use of relatively early items $(\mathrm{A}+/ \mathrm{C}-$ and $\mathrm{B}+/ \mathrm{E}-)$ and later items $(\mathrm{D}+/ \mathrm{F}-$ and $\mathrm{C}+/ \mathrm{F}-)$. To ensure that performance did not rely on the presence of a food reward, no food reward was initially placed in the pot for one of the three daily test trials after the initial stages of acquisition, again counterbalanced over days across the different temporal separations used; on these trials, the reward was placed on the sand immediately after the correct choice.

Before surgery, rats were required to reach a criterion of $80 \%$ correct performance for each lag for at least 10 successive trials. Once this criterion was reached, pairs of rats were randomly allocated to one of the two groups (AT and SHAM) on the basis of comparable performance for each lag. After a 2 week recovery period, all rats were trained using the same procedure for 20 trials at each lag. Values obtained on the last $10 \mathrm{~d}$ for preoperative and postoperative testing periods were used to evaluate performance. The experimenter was blind to group status for both the memory for temporal order and the recognition memory tasks.

Recognition memory tasks. To directly compare memory for the sequential order of events with memory for the prior occurrence of events independent of their order, the same rats were subsequently trained on two new successive tasks, requiring the recognition of individual items but not their order. These new tasks used exactly the same basic behavioral procedures as the memory for temporal order task and were conducted for $20 \mathrm{~d}$ (60 trials) each. A sample phase was used exactly as before, but for the recognition tasks the test phase contrasted any one of the sample odors presented in the list with any other odor not presented in the list for that trial. The "new" odor for that trial was one of the remaining possible odors. For the first recognition memory task, the rat was now rewarded for choosing the sample odor and not the new odor (a matching-to-sample procedure). The second recognition task was the reversal of this first recognition task; during the test phase, the rat was 
now rewarded when choosing the new odor that had not been presented during the sample phase versus an odor that had been experienced during the sample phase (a nonmatching-to-sample procedure). Attention was paid to ensure that all six odor positions from the sample phase were included in the test phase an equal number of times. One control rat became unwell after the first recognition task and was not included in the final task.

\section{Histology}

At the completion of the experiment, all rats were transcardially perfused with cold saline followed by $4 \%$ formalin. The brains were removed and postfixed for $2 \mathrm{~d}$ in $4 \%$ formalin, cryoprotected in $30 \%$ sucrose, and all the coronal $50 \mu \mathrm{m}$ sections throughout the thalamic region, obtained using a cryostat, were stained for cell bodies with cresyl violet. M.W. and J.C.D.-A., who were both blind to individual behavioral data, agreed on the lesion extent in each rat using the relevant plates of a rat brain atlas (Paxinos and Watson, 1998). We preferred to make a consensus of the lesions because inter-rater reliability across lesion cases does not ensure greater accuracy. The lesions were then replicated on electronic copies of the atlas and automated pixel counts of the relevant brain regions in this electronic version were used to generate estimated lesion volumes by factoring in the distances provided by the atlas. This procedure is an elaboration of the conventional "by eye" estimation used in the literature, but collapse of areas surrounding a lesion and variation in angle of sections required a conventional visual, rather than direct image, analysis. Acceptable lesions $(n=11)$ were defined as having $>50 \%$ bilateral damage to the AT nuclei, but not $>40 \%$ damage to either the adjacent lateral thalamic region (LT, comprising the rostral intralaminar nuclei and lateral mediodorsal thalamic nuclei) or the adjacent posteromedial thalamic region (MT, comprising the central and medial mediodorsal thalamic nuclei and the intermediodorsal nucleus). The reason for attending to these additional regions was that they have also been suggested as contributors to diencephalic amnesia (Mitchell and DalrympleAlford, 2005).

\section{Results}

\section{Histology}

Figure 1 depicts the largest and smallest acceptable AT lesions. Only rats with lesions meeting our criteria were included in the behavioral analyses (two exclusions had insufficient damage to the AT: 42.4 and $14.7 \%$ ). In the acceptable 11 AT rats there was a median of $80.2 \%$ damage (range, $57.3-96.1 \%$ ) to the AT nuclei, but only $6.9 \%$ damage (range, $2.3-29.3 \%$ ) to the LT region and $1.8 \%$ damage (range, $0.8-15.4 \%$ ) to the MT region. Median damage to other thalamic structures outside the AT nuclei was generally minimal to modest, with the exception of the interanteromedial nucleus in many but not all cases (27.2\%; range, $0.0-91.4 \%$ ): laterodorsal nucleus, $12.5 \%$ (range, $0.0-36.5 \%$ ); parataenial nucleus, $6.1 \%$ (range, $1.6-45.8 \%$ ); paraventricular and posterior paraventricular nuclei, $0.0 \%$; anterior paraventricular nucleus, $0.0 \%$; reuniens nucleus, $0.0 \%$; and rhomboid nuclei, $0.6 \%$ (range, $0.0-14.4 \%$ ).

\section{Memory for temporal order task: preoperative performance}

Rats that mastered the task before surgery took between 14-36 trials to reach a criterion of $80 \%$ correct responses over 10 consecutive trials on each lag (temporal separation). The average number of days $( \pm S D)$ to reach the criterion in these rats was $22.58 \pm 5.68$ for the 12 SHAM rats and $25.27 \pm 6.10$ for the $11 \mathrm{AT}$ rats, discounting the two excluded AT rats. The SHAM and AT rats received a total of $55 \mathrm{~d}$ of preoperative training, mainly because eight other rats $(\sim 25 \%)$ failed to learn the task adequately. These latter rats did not receive surgery but were run postoperatively to maintain identical housing and testing procedures.

An ANOVA conducted on performance of the SHAM and the AT groups for the last 10 preoperative days of testing, as a func-
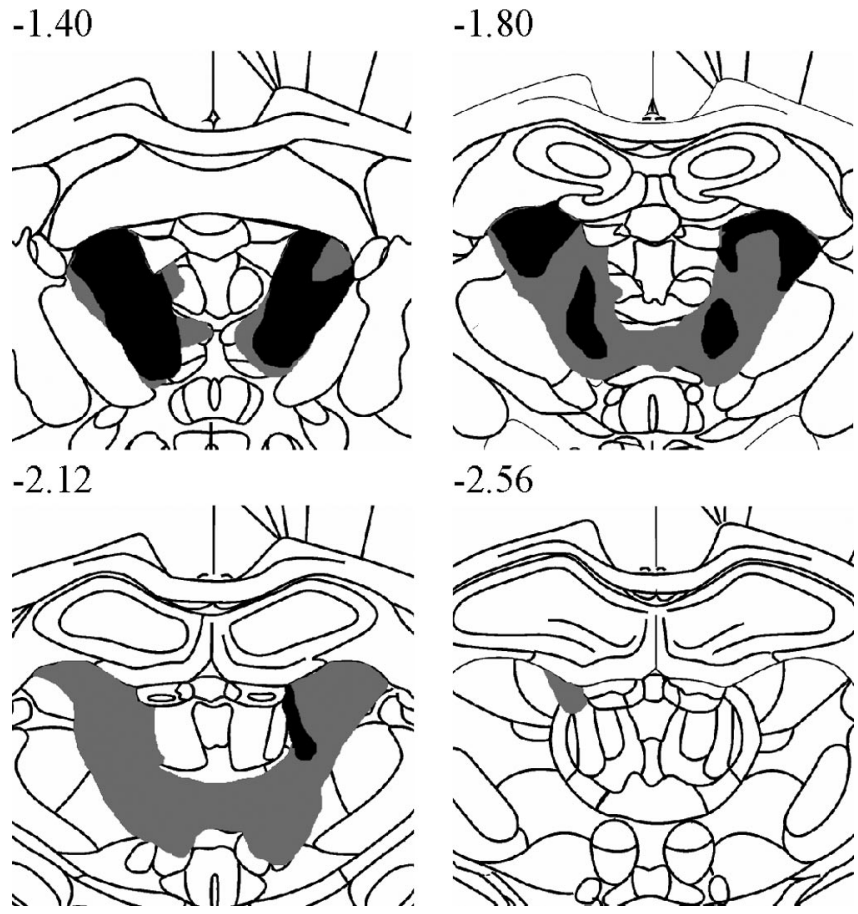

Figure 1. A series of coronal schematics throughout the medial thalamus showing the area of cell loss in the smallest (black) and largest (gray) AT lesion. Numbers refer to the distance from bregma (Paxinos and Watson, 1998). Note the minimal damage to thalamic nuclei outside the AT region (especially at -2.56 from bregma)

tion of temporal separation (lag 1,2, and 3 trials) and probe (that using odors involving early items in the sequence vs that using later items in the list), confirmed that there was no preoperative difference between groups $\left(F_{(1,21)}=1.28 ; p>0.25\right)$ or between probes that used relatively early versus later items in the list $\left(F_{(1,21)}=1.36 ; p>0.50\right)$. There was, however, a significant main effect of temporal separation $\left(F_{(2,42)}=4.65 ; p<0.02\right)$, with significantly superior performance for the lag 3 temporal separation versus the lag 1 temporal separation ( post hoc Fisher test; $p<$ $0.01)$ (Fig. 2A). There was no group by temporal separation interaction $\left(F_{(2,42)}=2.33 ; p>0.1\right)$. Preoperative test trials when the food reward was provided only after a digging response had been made compared with those trials when food was already buried in the sand indicated that performance was generally well above $80 \%$ at all lags, regardless of the presence of reward (before digging vs after digging: 89.6 and $82.6 \%$ for lag $1 ; 94.8$ and $87.8 \%$ for lag 2; 93.0 and $88.7 \%$ for lag 3), but the small mean difference between these two conditions was significant $\left(F_{(1,21)}=5.18 ; p<0.04\right)$.

Memory for temporal order task: postoperative performance The main finding in the current study was that a markedly different picture emerged for postsurgery testing on the temporal order task (Fig. $2 \mathrm{~B}$ ). ANOVA on the last $10 \mathrm{~d}$ of postoperative testing revealed a highly significant main effect of group $\left(F_{(1,21)}=\right.$ 77.46; $p<0.0001$ ), because the AT rats were now severely impaired relative to SHAM rats. Indeed, AT rats performed near chance levels for the memory for temporal order task, whereas SHAM rats continued at $\sim 80 \%$ correct performance on the test trials. After surgery, there was no main effect of temporal separation $\left(F_{(2,42)}=1.11 ; p>0.30\right)$ or probe type $\left(F_{(1,21)}=1.59 ; p>\right.$ $0.20)$. There were also no interaction effects [lag by early/late item probe and group by early/late item probe $(F s<1)$; group by lag by early/late item probe $\left.\left(F_{(2,42)}=2.10 ; p>0.1\right)\right]$. 

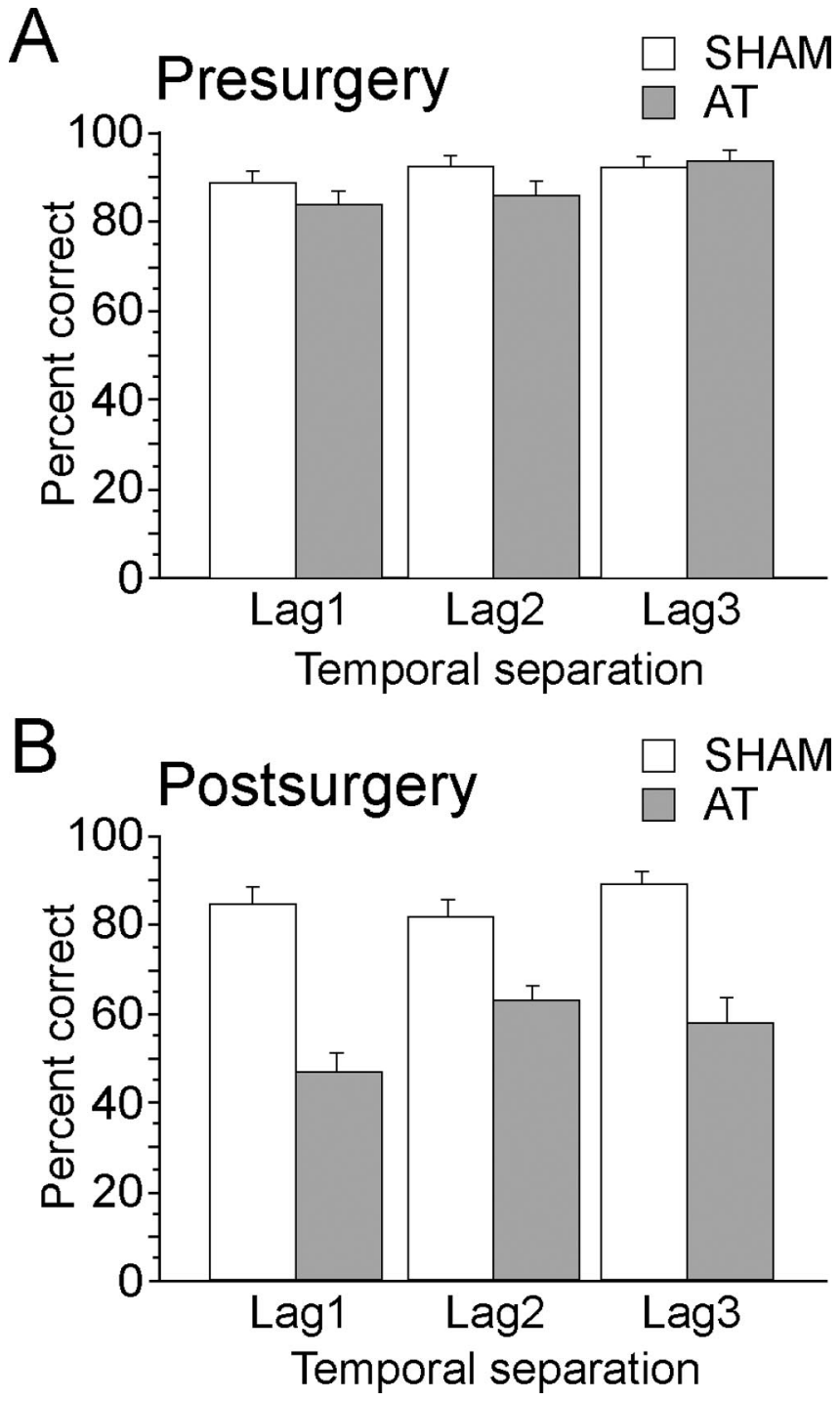

Figure 2. Mean ( \pm SEM) percentage of correct performance of AT and SHAM rats on the last $10 \mathrm{~d}$ of preoperative $(\boldsymbol{A})$ and postoperative $(\boldsymbol{B})$ training as a function of temporal separation. The lag number indicates the number of intermediate odors in the sample list that occurred between the two probe odors used in the test phase. The earlier item in the list was rewarded; list items varied pseudorandomly across trials, lag, and days of testing.

\section{First recognition memory task}

The first recognition task occurred immediately after completion of the main temporal order memory task. The rats were now required to distinguish between a recently experienced item (rewarded sample odor) from a nonsample odor (novel for that trial, but one of the 18 odors used daily). Figure $3 A$ shows the performance of SHAM and AT rats during the $20 \mathrm{~d}$ of testing on the first recognition task, expressed as four blocks of $5 \mathrm{~d}$ (i.e., 15 trials per block), which demonstrates the rate of acquisition on this new task. The two-way, repeated-measures ANOVA, with group (SHAM, AT) as a between-group factor and the successive blocks (blocks 1, 2, 3, and 4) as a within-group factor, revealed a significant main effect of group $\left(F_{(1,21)}=11.90 ; p<0.005\right)$ and block $\left(F_{(3,63)}=10.76 ; p<0.0001\right)$, but no group by block interaction $\left(F_{(3,63)}=1.34 ; p>0.20\right)$. Thus, although AT rats were impaired relative to SHAM rats for the first recognition task, both groups rapidly increased their level of performance across the successive blocks of testing. The performance of AT rats was above chance level for all blocks except the very first one $(p<0.0001$ for the last
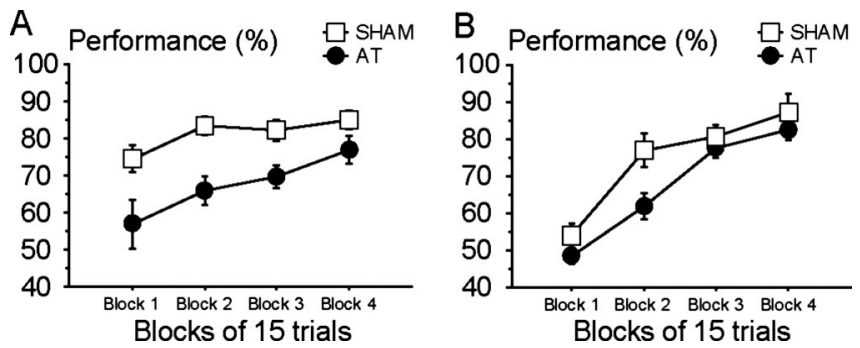

Figure 3. Mean ( \pm SEM) percentage of correct performance of AT and SHAM rats for the first recognition task $(\boldsymbol{A})$ (sample list item was rewarded and nonlist item was nonrewarded) and the second recognition task $(\boldsymbol{B})$ (reversal; sample list item was nonrewarded and the nonlist item was rewarded) as a function of blocks of trials. Each block represents $5 \mathrm{~d}$ of testing (i.e., 15 trials). Procedures were otherwise comparable with those used in the memory for temporal order task.

three blocks). Importantly, by the end of training on the first recognition memory task (last $5 \mathrm{~d}$ of testing), the AT rats had improved their performance to a final level of $77 \pm 12.4 \%$ correct and the SHAM rats reached $\sim 85 \pm 8.6 \%$ correct (Fig. $3 A$, last block). Separate analysis of these last $5 \mathrm{~d}$ of recognition memory testing revealed a nonsignificant difference between the two groups $\left(F_{(1,21)}=3.30 ; p<0.10\right)$, consistent with the view that the AT rats were able to perform well in this recognition memory task. To compare the effects of the six different probe trials used for this recognition task, the last $10 \mathrm{~d}$ of testing were evaluated, but this analysis produced no significant main effect of probes $\left(F_{(5,105)}<1\right)$ and the six different problems were similar for both groups of rats (group by probe interaction; $F_{(5,105)}<1$ ).

\section{Second recognition memory task-reversal}

The second recognition memory task was the reversal of the first recognition memory task and took place $9 \mathrm{~d}$ after the former. In the test phase of the second recognition memory task, the odor item presented during the sample phase was now the nonrewarded cue and it was the "new" odor (i.e., an odor that had not been presented in the sample phase for that trial) that was rewarded.

Both the SHAM and the AT groups acquired this new task rapidly. Figure $3 B$ depicts the performance of AT and SHAM rats for the second recognition task across four blocks of $5 \mathrm{~d}$ each (i.e., 15 trials per block). The two-way, repeated-measures ANOVA with group (SHAM, AT) as a between-group factor and the successive blocks (blocks 1, 2, 3, and 4) as a within-group factor again revealed a small but significant main effect of group $\left(F_{(1,20)}=\right.$ 4.76; $p<0.05)$ and a highly significant main effect of block $\left(F_{(3,60)}=46.96 ; p<0.0001\right)$, but no group by block interaction $\left(F_{(3,60)}=1.60 ; p>0.15\right)$. In this second recognition task, both groups exhibited an initial drop in performance to approximately chance levels for the first block of $5 \mathrm{~d}$, but SHAM rats and, slightly more slowly, AT rats quickly learned the new rule. Interestingly, only the first pair of days (six trials) of this first block of testing showed any evidence that the groups performed below chance $\left(t_{(21)}=4.12 ; p<0.001 ; 37.9 \%\right)$, whereas the groups showed above chance performance by the last $2 \mathrm{~d}$ (six trials) of the first block $\left(t_{(21)}=5.77 ; p<0.0001 ; 63.6 \%\right)$. ANOVA of the group by initial versus later pair of days of testing in the first block of $5 \mathrm{~d}$ of testing revealed no group effect $\left(F_{(1,20)}=2.34 ; p>0.13\right)$ and no interaction of group by initial versus later pair of days $\left(F_{(1,20)}<\right.$ $1)$, but there was a highly significant main effect of initial versus later pair of days $\left(F_{(1,20)}=56.11 ; p<0.0001\right)$. Clearly, both groups were capable of rapidly reversing their choice preference in this second recognition memory task. More importantly, the 


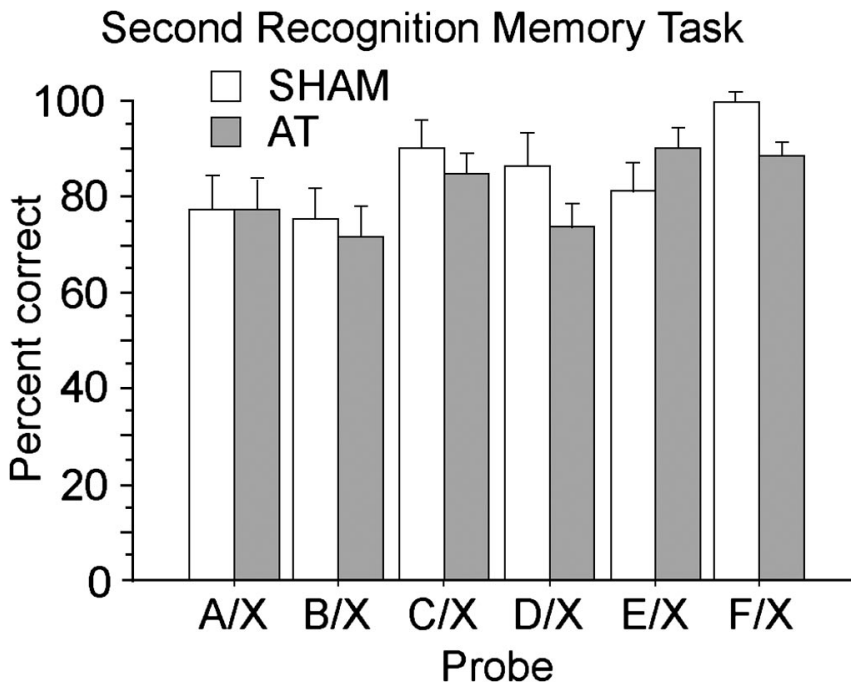

Figure 4. Mean ( \pm SEM) percentage of correct performance of AT and SHAM rats on the second recognition task for each of the different types of probe used. $X$ designates a randomly selected odor that was not presented in the sample list and was the rewarded odor in the test phase. The figure corresponds to the last $10 \mathrm{~d}$ of testing for the second recognition task; each probe had been tested five times.

data showed that the two groups achieved high levels of performance by the end of training (last $5 \mathrm{~d}$; SHAM $=90.9 \pm 15.6 \%$; $\mathrm{AT}=75.6 \pm 21.6 \%)$ and separate examination of this last block of testing revealed no evidence of a group effect at that stage $\left(F_{(1,20)}<1\right)$. Analysis of the last $10 \mathrm{~d}$ of training in the second recognition memory task was again used to determine the effects of the six probe types used. In this second recognition memory task, the probes involving the earlier items (A, B) from the list of sample odors were harder to discriminate relative to the new odor, whereas the last item was the easiest $\left(F_{(5,100)}=4.37 ; p<0.002\right)$. As shown in Figure 4 , this probe type effect was generally similar for both groups of rats (group by probe interaction; $F_{(5,100)}=1.28 ; p>0.20$ ).

\section{Comparison of final levels of performance across the three tasks}

To examine the final levels of performance across all three tasks, a two-way, repeated-measures ANOVA was conducted on mean performance for the last $5 \mathrm{~d}$ (last 15 trials) in all three tasks, with group (SHAM, AT) as the between-group factor and task as the within-group factor. In comparison to the corresponding data provided in the analysis of performance on the two recognition memory tasks, the mean level of performance at the end of postoperative testing on the temporal order task (last $5 \mathrm{~d}$, average on all kinds of lag) was SHAM $=80.6 \pm 6.3 \%$ and AT $=48.5$ $\pm 13.2 \%$. The ANOVA produced highly significant effects of group $\left(F_{(1,20)}=20.79 ; p<0.001\right)$, task $\left(F_{(2,40)}=24.45 ; p<\right.$ $0.0001)$, and group by task interaction $\left(F_{(2,20)}=11.10 ; p=\right.$ $0.0001)$. Whereas the SHAM group did not differ across tasks $\left(F_{(2,20)}=1.6 ; p>0.2\right)$, the AT group exhibited different levels of performance depending on the $\operatorname{task}\left(F_{(2,20)}=27.91 ; p<0.0001\right)$. In the AT group, performance was far superior at the very end of testing on both recognition memory tasks and, although these recognition memory tasks did not differ from each other, they contrasted markedly (Fisher test, $p<0.0001$ ) with the chance level of performance at the very end of postoperative training on the memory for temporal odor task.

\section{Discussion}

The present study showed that, despite extensive preoperative training, AT rats were severely impaired when required to recall the earlier cue in a list of odor items. This memory deficit was found across three temporal lags, with separations of one, two, and three intermediate items. In contrast, these AT rats were capable of acquiring two new tasks based on simple recognition memory and the prior occurrence of recent events independent of their order. Previous evidence on the influence of AT lesions on learning and memory in terms of its relationship to an extended hippocampal system has come exclusively from spatial memory tasks, especially allocentric spatial tasks requiring flexible responding and related tasks that depend on a spatial component (Parker and Gaffan, 1997; Aggleton and Brown, 1999; Sziklas and Petrides, 1999; van Groen et al., 2002; Mair et al., 2003; Moran and Dalrymple-Alford, 2003; Vann and Aggleton, 2004; Mitchell and Dalrymple-Alford, 2005). The current study provides the first unequivocal evidence to our knowledge of a detrimental effect of AT lesions on a hippocampal-dependent memory task that is independent of any spatial or related contextual memory requirements.

A previous study on memory for temporal order used only two objects as cues, spontaneous recognition procedures, and a long delay $(1 \mathrm{~h})$ between the two items and before the final test (Mitchell and Dalrymple-Alford, 2005). In that study, AT lesions produced no effect and it was the adjacent LT and MT lesions that tended to decrease performance on temporal order memory. In contrast, the current study used a large number of related items presented as a list of related events, a short interitem interval [ 3 min in our case; 2.5 min in Fortin et al. (2002); 3 s in Kesner et al. (2002)], and rewarded odor cues, so one or more of these factors is responsible for revealing deficits on memory for temporal order after AT lesions. The fact that the AT lesions used in the current study were highly selective, with minimal or little LT and MT damage, confirms the specific role of the AT nuclei when closely related events underpin memory for temporal order. It would be informative for future work to determine the effects of LT and MT lesions on the kind of temporal separation task that was used in the current report. The LT and MT regions have stronger connections with the medial prefrontal cortex (mPFC), which also appears to be important for memory for temporal order, at least in terms of the spontaneous two-object temporal order task (Mitchell and Laiacona, 1998; Hannesson et al., 2004). It is possible that $\mathrm{mPFC}$ lesions affect memory for temporal order regardless of the number of items and interitem delay, whereas hippocampal and AT lesions only affect temporal separation for numerous, closely linked items. Although the apparently similar deficits on memory for temporal order observed after both AT and hippocampal lesions may be attributable to their strong connections via the fornix, mamillary bodies, retrosplenial cortex, and subicular regions (Wyss and Van Groen, 1992; Shibata, 1993; Van Groen and Wyss, 1995; Shibata, 1998; van Groen et al., 1999), interconnections also exist between the AT nuclei and the mPFC (van Groen et al., 1999; Shibata and Naito, 2005), so the AT lesion effect on memory for temporal order in the current study may reflect both AT-hippocampal and AT-mPFC interactions. Future work on the effects of crossed unilateral hippocampal, mPFC, and AT lesions on memory for temporal order using the procedures used in the current study may help answer this question.

The initial performance of intact rats in the current study improved as a function of increased temporal distance between 
the two odors, which illustrates a graded pattern separation effect, but this overall relationship was relatively weak and did not extend to postoperative testing. The magnitude of this graded effect has, however, been at best modest in previous work on similar tasks, where it may have been increased by the forced use of only one probe for the largest separation, which is the two extreme items $(\mathrm{A}+/ \mathrm{E}-)$ when only five items occur in the sample list (Fortin et al., 2002; Kesner et al., 2002).

The pattern of results reported here makes it unlikely that other factors such as problems with basic recognition memory, primary sensory or discrimination deficits, lack of behavioral inhibition or poor behavioral flexibility, and strength of memory trace or task difficulty provide better explanations for the inability of the AT rats to remember the sequential order of a series of odors. Although rats with AT lesions were significantly poorer than SHAM rats during acquisition of the two recognition memory tasks, they learned them rapidly and were comparable with the SHAM group by the end of training on both tasks. The poor performance of the AT rats at the end of testing for memory of temporal order contrasted with both the rate of acquisition and final performance on the initial recognition memory task. Thus, it is unlikely that the differential performance was caused by a general recovery of function and, at least for spatial memory, deficits generally persist long-term in AT rats (van Groen et al., 2002; Moran and Dalrymple-Alford, 2003), even when pretraining is given before surgery (Warburton et al., 1999). The two recognition memory tasks also showed that the AT rats were able to make odor discriminations with trial-unique odor cues and to inhibit responding and demonstrate adequate behavioral flexibility. The second recognition memory task was one used by Fortin et al. (2002) and, as in that study, a temporal gradient for recognition memory was evident with performance relatively stronger for the more recently presented items. The fact that the temporal gradient effect for this recognition memory task did not differ markedly as a function of lesion status, yet memory for temporal order was impaired across all items in the list, also mimics their findings with hippocampal lesions. Similarly, task difficulty also did not explain the different effects of AT lesions across tasks (compare groups on the difficult $\mathrm{A} / \mathrm{X}$ and $\mathrm{B} / \mathrm{X}$ probes in Fig. 4 with performance across lags in Fig. $2 B$ ).

The question of whether spatial memory tasks sample a unique type of memory or often provide examples of a more general characteristic, such as declarative or episodic memory, has been a discussion point for more than 25 years (O'Keefe and Nadel, 1978; O'Keefe, 1991; Eichenbaum, 2000; Aggleton and Pearce, 2001). It has been suggested that the influence of hippocampal lesions has more to do with memory space and the flexible use of overlapping representations of closely linked events rather than specifically spatial memory (Eichenbaum et al., 1999). An alternative view is that spatial memory is one important attribute for which the hippocampal system is responsible and that memory for temporal attributes constitutes an equally important but separate function of the hippocampus (Kesner and Rogers, 2004). That hippocampal lesions produce deficits in memory for temporal order is one of the strongest pieces of evidence in favor of the notion that the hippocampus contributes to memory that extends beyond the spatial domain. Initially, this human and animal evidence used memory for the temporal order of spatial locations (Chiba et al., 1994; Hopkins et al., 1995; Chiba et al., 1997), but this caveat is not an issue in the two independent reports that hippocampal lesions also disrupted memory for the temporal order of a list of odors in nonspatial tasks (Fortin et al., 2002; Kesner et al., 2002). Knowledge that AT lesions also produce profound deficits in nonspatial memory for temporal order reinforces the idea that this brain region is part of an extended hippocampal system (Aggleton and Brown, 1999) and, like the hippocampal system, has an influence beyond spatial memory. Just as the severity of the effects of large AT lesions on spatial memory tasks is often comparable with that of hippocampal lesions and sometimes, more extensive than that, of fornix lesions (Sziklas and Petrides, 1999; Warburton and Aggleton, 1999; Sziklas and Petrides, 2002), the close concordance of the pattern of results in the current study after AT lesions to that after hippocampal lesions (Fortin et al., 2002) adds weight to the suggested functional interdependence of the two structures. This interdependence is reinforced by evidence, albeit from spatial tasks, that crossed unilateral lesions of the AT nuclei and the hippocampal system can produce severe memory deficits and that AT lesions produce reduced neuronal activity in the limbic system, including the retrosplenial cortex and hippocampus (Warburton et al., 2001; Jenkins et al., 2002; Jenkins et al., 2004). It would be valuable to have similar crossed-lesion and neuronalactivation evidence from the context of memory for temporal order, as well as tasks that examine memory for the frequency of events and memory for duration, which have also been reported as sensitive to hippocampal lesions (Kesner, 1990; Jackson et al., 1998; Kesner, 1998). In conclusion, AT lesions produce substantial deficits when the memory requirement is demanding, in terms of either spatial or temporal context. This pattern of deficits supports the proposed functional interdependence of AThippocampal system connections.

\section{References}

Aggleton JP, Brown MW (1999) Episodic memory, amnesia, and the hippocampal-anterior thalamic axis. Behav Brain Sci 22:425-444; discussion 444-489.

Aggleton JP, Pearce JM (2001) Neural systems underlying episodic memory: insights from animal research. Philos Trans R Soc Lond B Biol Sci 356:1467-1482.

Aggleton JP, Hunt PR, Nagle S, Neave N (1996) The effects of selective lesions within the anterior thalamic nuclei on spatial memory in the rat. Behav Brain Res 81:189-198.

Beracochea DJ, Jaffard R, Jarrard LE (1989) Effects of anterior or dorsomedial thalamic ibotenic lesions on learning and memory in rats. Behav Neural Biol 51:364-376.

Byatt G, Dalrymple-Alford JC (1996) Both anteromedial and anteroventral thalamic lesions impair radial-maze learning in rats. Behav Neurosci 110:1335-1348.

Celerier A, Ognard R, Decorte L, Beracochea D (2000) Deficits of spatial and nonspatial memory and of auditory fear conditioning following anterior thalamic lesions in mice: comparison with chronic alcohol consumption. Eur J Neurosci 12:2575-2584.

Chiba AA, Kesner RP, Reynolds AM (1994) Memory for spatial location as a function of temporal lag in rats: role of hippocampus and medial prefrontal cortex. Behav Neural Biol 61:123-131.

Chiba AA, Kesner RP, Gibson CJ (1997) Memory for temporal order of new and familiar spatial location sequences: role of the medial prefrontal cortex. Learn Mem 4:311-317.

Dudchenko PA, Wood ER, Eichenbaum H (2000) Neurotoxic hippocampal lesions have no effect on odor span and little effect on odor recognition memory but produce significant impairments on spatial span, recognition, and alternation. J Neurosci 20:2964-2977.

Eichenbaum H (2000) A cortical-hippocampal system for declarative memory. Nat Rev Neurosci 1:41-50.

Eichenbaum H, Dudchenko P, Wood E, Shapiro M, Tanila H (1999) The hippocampus, memory, and place cells: is it spatial memory or memory space? Neuron 23:209-226.

Fortin NJ, Agster KL, Eichenbaum HB (2002) Critical role of the hippocampus in memory for sequences of events. Nat Neurosci 5:458-462.

Foster JK, Rawlins JN (1992) Hippocampal aspiration lesions fail to impair 
performance of a delayed alternation GO/NO-GO task. Behav Brain Res 47:35-48.

Hannesson DK, Vacca G, Howland JG, Phillips AG (2004) Medial prefrontal cortex is involved in spatial temporal order memory but not spatial recognition memory in tests relying on spontaneous exploration in rats. Behav Brain Res 153:273-285.

Harding A, Halliday G, Caine D, Kril J (2000) Degeneration of anterior thalamic nuclei differentiates alcoholics with amnesia Brain $123(\mathrm{Pt}$ 1):141-154.

Hopkins RO, Kesner RP, Goldstein M (1995) Item and order recognition memory in subjects with hypoxic brain injury. Brain Cogn 27:180-201.

Jackson PA, Kesner RP, Amann K (1998) Memory for duration: role of hippocampus and medial prefrontal cortex. Neurobiol Learn Mem 70:328-348.

Jenkins TA, Dias R, Amin E, Brown MW, Aggleton JP (2002) Fos imaging reveals that lesions of the anterior thalamic nuclei produce widespread limbic hypoactivity in rats. J Neurosci 22:5230-5238.

Jenkins TA, Vann SD, Amin E, Aggleton JP (2004) Anterior thalamic lesions stop immediate early gene activation in selective laminae of the retrosplenial cortex: evidence of covert pathology in rats? Eur J Neurosci 19:3291-3304.

Kesner RP (1990) Memory for frequency in rats: role of the hippocampus and medial prefrontal cortex. Behav Neural Biol 53:402-410.

Kesner RP (1998) Neural mediation of memory for time: role of the hippocampus and medial prefrontal cortex. Psychon Bull Rev 5:585-596.

Kesner RP, Rogers J (2004) An analysis of independence and interactions of brain substrates that subserve multiple attributes, memory systems, and underlying processes. Neurobiol Learn Mem 82:199-215.

Kesner RP, Gilbert PE, Barua LA (2002) The role of the hippocampus in memory for the temporal order of a sequence of odors. Behav Neurosci 116:286-290.

Mair RG, Burk JA, Porter MC (2003) Impairment of radial maze delayed nonmatching after lesions of anterior thalamus and parahippocampal cortex. Behav Neurosci 117:596-605.

Mitchell AS, Dalrymple-Alford JC (2005) Dissociable memory effects after medial thalamus lesions in the rat. Eur J Neurosci 22:973-985.

Mitchell JB, Laiacona J (1998) The medial frontal cortex and temporal memory: tests using spontaneous exploratory behaviour in the rat. Behav Brain Res 97:107-113.

Moran JP, Dalrymple-Alford JC (2003) Perirhinal cortex and anterior thalamic lesions: comparative effects on learning and memory. Behav Neurosci 117:1326-1341.

O'Keefe J (1991) An allocentric spatial model for the hippocampal cognitive map. Hippocampus 1:230-235.

O'Keefe J, Nadel L (1978) The hippocampus as a cognitive map. London: Oxford UP.

Parker A, Gaffan D (1997) The effect of anterior thalamic and cingulate cortex lesions on object-in-place memory in monkeys. Neuropsychologia 35:1093-1102.
Paxinos G, Watson C (1998) The rat brain in stereotaxic coordinates, Ed 4 San Diego: Academic, Harcourt Brace.

Peinado-Manzano MA, Pozo-Garcia R (1991) The role of different nuclei of the thalamus in processing episodic information. Behav Brain Res 45:17-27.

Shibata H (1993) Direct projections from the anterior thalamic nuclei to the retrohippocampal region in the rat. J Comp Neurol 337:431-445.

Shibata H (1998) Organization of projections of rat retrosplenial cortex to the anterior thalamic nuclei. Eur J Neurosci 10:3210-3219.

Shibata H, Naito J (2005) Organization of anterior cingulate and frontal cortical projections to the anterior and laterodorsal thalamic nuclei in the rat. Brain Res 1059:93-103.

Sziklas V, Petrides M (1999) The effects of lesions to the anterior thalamic nuclei on object-place associations in rats. Eur J Neurosci 11:559-566.

Sziklas V, Petrides M (2002) Effects of lesions to the hippocampus or the fornix on allocentric conditional associative learning in rats. Hippocampus 12:543-550.

Van der Werf YD, Witter MP, Uylings HB, Jolles J (2000) Neuropsychology of infarctions in the thalamus: a review. Neuropsychologia 38:613-627.

Van Groen T, Wyss JM (1995) Projections from the anterodorsal and anteroventral nucleus of the thalamus to the limbic cortex in the rat. J Comp Neurol 358:584-604.

van Groen T, Kadish I, Wyss JM (1999) Efferent connections of the anteromedial nucleus of the thalamus of the rat. Brain Res Brain Res Rev 30:1-26.

van Groen T, Kadish I, Michael Wyss J (2002) Role of the anterodorsal and anteroventral nuclei of the thalamus in spatial memory in the rat. Behav Brain Res 132:19-28.

Vann SD, Aggleton JP (2004) The mammillary bodies: two memory systems in one? Nat Rev Neurosci 5:35-44.

Warburton EC, Aggleton JP (1999) Differential deficits in the Morris water maze following cytotoxic lesions of the anterior thalamus and fornix transection. Behav Brain Res 98:27-38.

Warburton EC, Morgan A, Baird AL, Muir JL, Aggleton JP (1999) Does pretraining spare the spatial deficit associated with anterior thalamic damage in rats? Behav Neurosci 113:956-967.

Warburton EC, Baird A, Morgan A, Muir JL, Aggleton JP (2001) The conjoint importance of the hippocampus and anterior thalamic nuclei for allocentric spatial learning: Evidence from a disconnection study in the rat. J Neurosci 21:7323-7330.

Ward-Robinson J, Wilton LAK, Muir RC, Honey SD, Vann SD, Aggleton JP (2002) Sensory preconditioning in rats with lesions of the anterior thalamic nuclei: evidence for intact nonspatial "relational" processing. Behav Brain Res 133:125-133.

Winocur G (1985) The hippocampus and thalamus: their roles in short- and long-term memory and the effects of interference. Behav Brain Res 16:135-152.

Wyss JM, Van Groen T (1992) Connections between the retrosplenial cortex and the hippocampal formation in the rat: a review. Hippocampus $2: 1-11$. 